PROCEEDINGS OF THE

AMERICAN MATHEMATICAL SOCIETY

Volume 134, Number 1, Pages 31-37

S 0002-9939(05)08292-4

Article electronically published on August 11, 2005

\title{
A NOTE ON SELMER GROUPS OF ABELIAN VARIETIES OVER THE TRIVIALIZING EXTENSIONS
}

\author{
YOSHIHIRO OCHI \\ (Communicated by David E. Rohrlich)
}

\begin{abstract}
We prove that for any abelian variety $A$ defined over a number field $K$ that is not isogenous to a product of CM elliptic curves, the pontrjagin dual of the Selmer group of the abelian variety over the trivializing extension $K\left(A\left[p^{\infty}\right]\right)$ has no nonzero pseudo-null submodules.
\end{abstract}

The present paper can be viewed as a generalization to abelian varieties of the following

Theorem (Perrin-Riou, Ochi-Venjakob). Let $E$ be an elliptic curve over a number field $K$, and let $p$ be an odd prime number. Let $K_{\infty}$ denote $K\left(E\left[p^{\infty}\right]\right)$ and let $G=\operatorname{Gal}\left(K_{\infty} / K\right)$. Then the pontrjagin dual of the p-primary Selmer group over $K_{\infty}$ has no nonzero pseudo-null $\mathbb{Z}_{p}[[G]]$-submodules.

The CM case was proved by B. Perrin-Riou ([ $[\mathrm{Pe}]$, Theorem 2.4) and the nonCM case was proved in OV1. The similar problems for the case of $\mathbb{Z}_{p}$-extensions were worked out by R. Greenberg $([\mathrm{Gr}]$ ) and by Y. Hachimori and K. Matsuno (see Ma $)$. In this paper we shall generalize it to abelian varieties that are not isogenous to a product of CM elliptic curves.

Let $A$ be an abelian variety defined over a number field $K$. We fix an odd prime number $p>2$. $A\left[p^{n}\right]$ denotes $\operatorname{Ker}\left(A(\overline{\mathbb{Q}}) \stackrel{p^{n}}{\rightarrow} A(\overline{\mathbb{Q}})\right)$ and $A(p):=\bigcup_{n=1}^{\infty} A\left[p^{n}\right]$. They are $G_{K}$-modules, where $G_{K}:=\operatorname{Gal}(\bar{K} / K)$. We consider a tower of extensions $K_{n}:=K\left(A\left[p^{n+1}\right]\right)$ and $K_{\infty}:=\bigcup_{n=1}^{\infty} K_{n}$. Throughout this paper $G$ will denote the Galois group $\operatorname{Gal}(K(A(p)) / K)$, unless otherwise mentioned. The Iwasawa algebra of $G$ is $\Lambda:=\Lambda(G)=\lim _{\longleftarrow} \mathbb{Z}_{p}[G / U]$, where $U$ runs over all open normal subgroups of $G$.

The ( $p$-primary) Selmer group is defined as follows:

$$
\operatorname{Sel}_{p^{\infty}}(A / K):=\operatorname{Ker}\left(H^{1}(K, A(p)) \rightarrow \prod_{v} H^{1}\left(K_{v}, A\right)\right) .
$$

The Selmer group $\operatorname{Sel}_{p} \infty\left(A / K_{n}\right)$ over the field $K_{n}$ is defined in the same way, and $\operatorname{Sel}_{p^{\infty}}\left(A / K_{\infty}\right)$ is defined by $\lim _{\longrightarrow} \operatorname{Sel}_{p^{\infty}}\left(A / K_{n}\right)$. This has the natural structure of a $\Lambda(G)$-module.

Received by the editors August 11, 2004.

2000 Mathematics Subject Classification. Primary 11R23, 11G10.

Key words and phrases. Selmer groups, abelian varieties, Iwasawa theory.

(C)2005 American Mathematical Society Reverts to public domain 28 years from publication 
Assume $A$ has good reduction at any prime over $p$. Let $S$ be a finite set of places of $K$ containing all primes above $p$ and primes where $A$ has bad reduction. Coates and Greenberg ([CG]) have established an isomorphism

$$
H^{1}\left(K_{v, \infty}, A\right)(p) \cong H^{1}\left(K_{v, \infty}, \widetilde{A}(p)\right)
$$

where $v$ is a prime above $p$ and $\widetilde{A}$ denotes $\bmod -v$ reduction of the abelian variety $A$, which is an abelian variety defined over a finite field by the assumption. Therefore, defining $\widetilde{A}(p)=A(p)$ for any $v \nmid p$, we have the following exact sequence of $\Lambda(G)$ modules:

$$
0 \rightarrow \operatorname{Sel}_{p^{\infty}}\left(A / K_{\infty}\right) \rightarrow H^{1}\left(K_{S} / K_{\infty}, A(p)\right) \stackrel{\psi}{\rightarrow} \bigoplus_{S} \operatorname{Coind}_{G_{v}}^{G} H^{1}\left(K_{v, \infty}, \widetilde{A}(p)\right) .
$$

In this paper we always assume the following:

(1) $A$ has good reduction at any prime over $p$.

(2) The map $\psi$ in the sequence (2) is surjective.

(3) $A$ is not isogenous to a product of CM elliptic curves.

There is a fundamental conjecture on the $\Lambda$-rank of the pontrjagin dual of the Selmer group $\mathfrak{X}:=\operatorname{Sel}_{p^{\infty}}\left(A / K_{\infty}\right)^{\vee}$ (see, for instance, conjecture 7.4 of [OV2]). It is known that the conjecture implies the second assumption.

Under these assumptions we prove the following

Theorem. Let $A$ be an abelian variety defined over a number field $K$. Let $p$ be an odd prime. Assume the three assumptions (1), (2) and (3) stated above. Let $K_{\infty}=K(A(p))$ and $G=\operatorname{Gal}\left(K_{\infty} / K\right)$. Then the pontrjagin dual of the Selmer group $\mathfrak{X}=\operatorname{Sel}_{p^{\infty}}\left(A / K_{\infty}\right)^{\vee}$ does not have nontrivial pseudo-null $\Lambda(G)$-submodules.

The reason why we need assumption (3) is that our proof requires the dimension of $G$ as a $p$-adic Lie group to be greater than 2 , which is the case with the abelian varieties satisfying assumption (3), as the following lemma implies. The proof of the lemma is due to Yuichiro Taguchi.

Lemma 1. Assume $A$ is an abelian variety over $K$ of dimension $>1$ and simple over $\bar{K}$. Then the dimension of $G$ as a $p$-adic Lie group is greater than or equal to 3.

Proof. Denote Zariski closure of $G$ in $G L_{2 g}\left(\mathbb{Q}_{p}\right)$ by $Z$, where $g=\operatorname{dim}(A)$. By a theorem of Bogomolov ( $[\mathrm{Se}$, p. 3$), G$ is open in $Z\left(\mathbb{Q}_{p}\right)$. It is also known that $Z$ contains a two-dimensional torus ( $\mathrm{Se}$, p. 11). Hence if $G$ is not potentially abelian, we get $\operatorname{dim}(G) \geq 3$. If $G$ is potentially abelian, then $A$ is of CM type, and according to Ribet $([\mathrm{Ri}])$, we have $\operatorname{dim}(G) \geq 3$.

By assumption (3) and Lemma 1, we have $\operatorname{dim}(G) \geq 3$, because if $A$ contains a simple abelian subvariety $B$ which has dimension greater than 1, then by Lemma 11. we have $\operatorname{dim}(G)>2$. If $A$ is isogenous to a product of elliptic curves, at least one of which is an elliptic curve without CM, then we have $\operatorname{dim}(G)>2$, by a theorem of Serre which says the dimension of $G$ as a $p$-adic Lie group is equal to 4 for any elliptic curve that has no CM.

The definition of a pseudo-null submodule for a commutative noetherian ring is well known: For such a ring $R$, a finitely generated $R$-module $M$ is pseudo-null if the Krull dimension of $M$ is less than $\operatorname{dim}(R)-1$. This notion has been extended to the cases of noncommutative rings, including the Iwasawa algebra $\Lambda=\mathbb{Z}_{p}[[G]]$ 
by Venjakob $([\mathrm{Ve}])$. We write $\mathrm{E}^{i}(M):=\operatorname{Ext}_{\Lambda}^{i}(M, \Lambda)$ for a $\Lambda$-module $M$. Here we just recall that for any finitely generated $\Lambda$-module $M$ :

- $M$ is a pseudo-null module if and only if $E^{0}(M)=\mathrm{E}^{1}(M)=0$.

- $M$ does not have any nonzero pseudo-null submodule if $\mathrm{E}^{i} \mathrm{E}^{i}(M)=0$ for all $i \geq 2$.

The Pontrjagin dual of a $\mathbb{Z}_{p^{-}}$-module $M$, denoted by $M^{\vee}$, is defined as

$$
M^{\vee}:=\operatorname{Hom}_{\mathbb{Z}_{p}, \text { cont }}\left(M, \mathbb{Q}_{p} / \mathbb{Z}_{p}\right) .
$$

Denote by $\Omega$ the maximal pro- $p$ extension of $K_{\infty}$ unramified outside $S$. Put $\mathcal{H}=$ $G\left(\Omega / K_{\infty}\right)$ and $\mathcal{G}=G(\Omega / K)$. The ideal $I(\mathcal{G})$ is defined as the kernel of the natural map $\Lambda(\mathcal{G}) \rightarrow \mathbb{Z}_{p}$ so that $0 \rightarrow I(\mathcal{G}) \rightarrow \Lambda(\mathcal{G}) \rightarrow \mathbb{Z}_{p} \rightarrow 0$ is exact. For a finitely generated $\Lambda(\mathcal{G})$-module $M$, we define $M[A(p)]:=\operatorname{Hom}_{\mathbb{Z}_{p}, \text { cont }}(M, A(p))^{\vee}=M \otimes_{\mathbb{Z}_{p}}$ $A(p)^{\vee}$. In the paper OV1 we have proved the following things:

- $Y:=(I(\mathcal{G})[A(p)])_{\mathcal{H}}$ has projective dimension $\leq 1$.

- There is the following short exact sequence of finitely generated $\Lambda$-modules:

$$
0 \rightarrow X \rightarrow Y \rightarrow J \rightarrow 0,
$$

where $J:=\operatorname{Ker}\left((\Lambda(\mathcal{G})[A(p)])_{\mathcal{H}} \rightarrow A(p)^{\vee}\right)$ and $X:=H^{1}\left(K_{S} / K_{\infty}, A(p)\right)^{\vee}$.

We may assume $\operatorname{dim}(A) \geq 2$. We may take $K$ such that $K=K_{0}$, and $S=\{v$ : $v \mid p\}$. The reasons are the same as in the one-dimensional case (see OV1]). We can take $K=K_{0}$ because our aim is to show the vanishing of some Ext-groups and there is the following isomorphism ([Ja, 2.3$)$ :

$$
\operatorname{Ext}_{\Lambda(G)}^{i}(M, \Lambda(G)) \cong \operatorname{Ext}_{\Lambda(U)}^{i}(M, \Lambda(U))
$$

for all $i \geq 0, U$ any open subgroup of $G$ and $M$ any finitely generated $\Lambda(G)$-module. It is easy to see that if $v \nmid p$ and $\operatorname{dim}\left(G\left(K_{v}(A(p)) / K_{v}\right)\right)=1$, then $A$ has potentially good reduction by Ogg-Shafarevich's criterion ([ST]). By a result of Serre-Tate in [ST, there is no such place in $K_{0}(\underline{\mathrm{ST}}, 2-2)$. If $v \nmid p$ and $\operatorname{dim}\left(G\left(K_{v}(A(p)) / K_{v}\right)\right)>$ 1 , we have $H^{1}\left(K_{v}, A\right)(p)=H^{1}\left(K_{v, \infty}, A(p)\right)=0$ by 5.4 of [OV1]. So if we have $K=K_{0}$, then we can take our $S$ to be the set $\{v: v \mid p\}$.

Write $X_{v}=H^{1}\left(K_{v, \infty}, \widetilde{A}(p)\right)^{\vee}, X=H^{1}\left(K_{S} / K_{\infty}, A(p)\right)^{\vee}$ and $\mathfrak{X}=\operatorname{Sel}_{p \infty}\left(A / K_{\infty}\right)^{\vee}$. By assumption, we have the following exact sequence:

$$
0 \rightarrow \bigoplus_{S} \operatorname{Ind}_{G}^{G_{v}} X_{v} \rightarrow X \rightarrow \mathfrak{X} \rightarrow 0
$$

We often use the facts that $\mathrm{E}^{1}\left(\operatorname{Ind}_{G}^{G_{v}} X_{v}\right)=\operatorname{Ind}_{G}^{G_{v}} \mathrm{E}_{\Lambda\left(G_{v}\right)}^{1}\left(X_{v}\right)(\underline{\mathrm{OV} 1}, 5.5)$, and that $E^{i}(X)=\mathrm{E}^{i+1}(J)=\mathrm{E}^{i+2}\left(A(p)^{\vee}\right)$ for all $i \geq 2$ (the latter follows from the fact that $Y$ has projective dimension $\leq 1$ and the definition of $J)$. Put $\mathrm{E}_{v}^{i}(M):=$ $\operatorname{Ext}_{\Lambda\left(G_{v}\right)}^{i}\left(M, \Lambda\left(G_{v}\right)\right)$.

Lemma 2. Let $G$ be a p-adic Lie group without p-torsion elements of dimension d, and let $N$ be a $\Lambda(G)$-module. Assume that $N$ is finitely generated as a $\mathbb{Z}_{p}$-module. Then:

(1) If $N$ is $\mathbb{Z}_{p}$-free, then $E^{i}(N)=0$ for $i \neq d$ and $E^{d}(N)$ is torsion free. If $N$ is finite, then $E^{i}(N)=0$ for $i \neq d+1$ and $E^{d+1}(N)$ is finite.

(2) For the $X$ defined above, $E^{i}(X)=0$ if $i \neq 0,1, d-2$.

(3) $E_{v}^{i} E_{v}^{i-1}\left(X_{v}\right)=0$ for $i \geq 3$. 
Proof. The first one is a result of U. Jannsen ( $[\mathrm{Ja}, 2.6)$. The second one follows from the first one since $E^{i}(X)=\mathrm{E}^{i+2}\left(A(p)^{\vee}\right)$ (for $i \geq 2$ ). The third one follows from the first one and the local version of the sequence (3).

To prove the theorem, we show $\mathrm{E}^{i} \mathrm{E}^{i}(\mathfrak{X})=0$ for all $i \geq 2$. Recall that there is the exact sequence $0 \rightarrow \bigoplus_{v \mid p} \operatorname{Ind}_{G}^{G_{v}} X_{v} \rightarrow X \rightarrow \mathfrak{X} \rightarrow 0$. This gives an exact sequence $\mathrm{E}^{i-1}(X) \rightarrow \bigoplus_{v \mid p} \operatorname{Ind}_{G}^{G_{v}} \mathrm{E}_{v}^{i-1}\left(X_{v}\right) \rightarrow \mathrm{E}^{i}(\mathfrak{X}) \rightarrow \mathrm{E}^{i}(X)$.

First we assume $i \geq 3$.

a) Suppose $\mathrm{E}^{i}(X)=\mathrm{E}^{i-1}(X)=0$. Then we have

$$
\mathrm{E}^{i} \mathrm{E}^{i}(\mathfrak{X})=\bigoplus \operatorname{Ind}_{G}^{G_{v}} \mathrm{E}_{v}^{i} \mathrm{E}_{v}^{i-1}\left(X_{v}\right)=0
$$

by Lemma 2 .

b) Suppose $\mathrm{E}^{i}(X) \neq 0$ but $\mathrm{E}^{i-1}(X)=0$. Then $i=d-2$. We have the exact sequence $\mathrm{E}^{i-1}(X)=0 \rightarrow \bigoplus \operatorname{Ind}_{G}^{G_{v}} \mathrm{E}_{v}^{i-1}\left(X_{v}\right) \rightarrow \mathrm{E}^{i}(\mathfrak{X}) \rightarrow N \rightarrow 0$ with $N$ a submodule of $\mathrm{E}^{i}(X)$. Since $N$ is a finitely generated $\mathbb{Z}_{p}$-module, we have $\mathrm{E}^{i}(N)=0 \rightarrow \mathrm{E}^{i} \mathrm{E}^{i}(\mathfrak{X}) \rightarrow \bigoplus \operatorname{Ind}_{G}^{G_{v}} \mathrm{E}_{v}^{i} \mathrm{E}_{v}^{i-1}\left(X_{v}\right)=0$ by Lemma 2

c) Suppose $\mathrm{E}^{i-1}(X) \neq 0$ but $\mathrm{E}^{i}(X)=0$. Then $i=d-1$ by Lemma 2, This time we have an exact sequence $0 \rightarrow N \rightarrow \bigoplus \operatorname{Ind}_{G}^{G_{v}} \mathrm{E}_{v}^{i-1}\left(X_{v}\right) \rightarrow \mathrm{E}^{i}(\mathfrak{X}) \rightarrow 0$ with $N$ a quotient of $\mathrm{E}^{i-1}(X)$, and $\mathrm{E}^{i-1}(N)=0 \rightarrow \mathrm{E}^{i} \mathrm{E}^{i}(\mathfrak{X}) \rightarrow \operatorname{Ind}_{G}^{G_{v}} \mathrm{E}_{v}^{i} \mathrm{E}_{v}^{i-1}\left(X_{v}\right)=0$ similarly. Hence we obtain $\mathrm{E}^{i} \mathrm{E}^{i}(\mathfrak{X})=0$.

Now let us consider the case $i=2$. Our aim is to show $\mathrm{E}^{2} \mathrm{E}^{2}(\mathfrak{X})=0$. Assume first that $\mathrm{E}^{2}(X)=0$. In this case $\operatorname{dim}(G) \neq 4$ by Lemma 2

Let us define $Y_{v}, J_{v}$ and $X_{v}$ similarly for each place $v$ of $K$ but by replacing $A(p)^{\vee}$ with $\widetilde{A}(p)^{\vee}$. For example $X_{v}:=H^{1}\left(K_{v, \infty}, \widetilde{A}(p)\right)^{\vee}$. As in the global case, we have an exact sequence $0 \rightarrow X_{v} \rightarrow Y_{v} \rightarrow J_{v} \rightarrow 0$ for each place $v$ of the number field.

Lemma 3. There is the following commutative diagram:

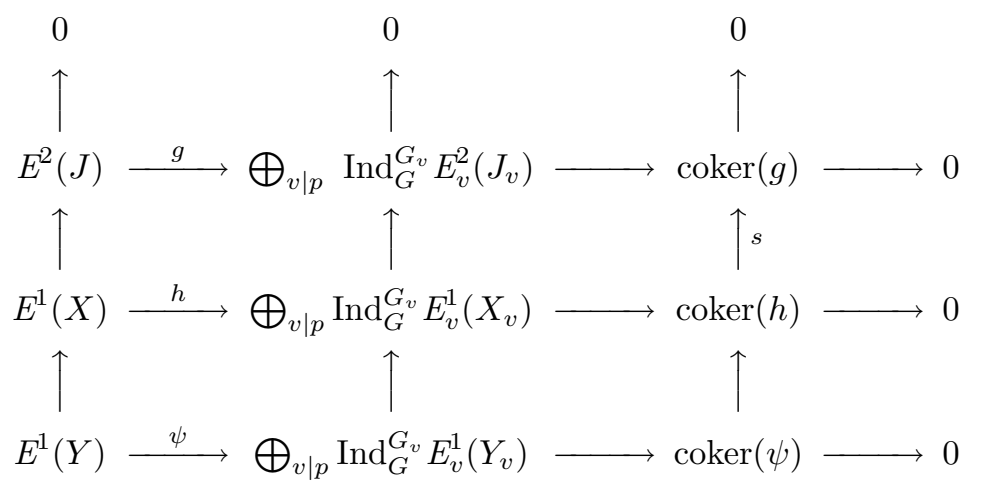

Proof. That $Y$ has projective dimension $\leq 1$ implies $E^{2}(Y)=0$, which explains the 0 at the top of the first column in the diagram. Let $\mathcal{G}_{v} \subset \mathcal{G}$ be a decomposition group. Then there are the natural maps $\Lambda\left(\mathcal{G}_{v}\right) \rightarrow \Lambda(\mathcal{G})$ and $I\left(\mathcal{G}_{v}\right) \rightarrow I(\mathcal{G})$. The map $A(p) \rightarrow \widetilde{A}(p)$ gives the dual $\widetilde{A}(p)^{\vee} \rightarrow A(p)^{\vee}$ and $I\left(\mathcal{G}_{v}\right) \otimes \widetilde{A}(p)^{\vee} \rightarrow I(\mathcal{G}) \otimes A(p)^{\vee}$. Let $\mathcal{H}_{v}=\mathcal{G}_{v} \cap \mathcal{H}$. Since in general $H \subset G$ gives the natural map $M_{H} \rightarrow M_{G}$, we have

$$
\left(I\left(\mathcal{G}_{v}\right) \otimes \widetilde{A}(p)^{\vee}\right)_{\mathcal{H}_{v}} \rightarrow\left(I(\mathcal{G}) \otimes A(p)^{\vee}\right)_{\mathcal{H}_{v}} \rightarrow\left(I(\mathcal{G}) \otimes A(p)^{\vee}\right)_{\mathcal{H}}
$$


Hence we get $\varphi: \operatorname{Ind}_{G}^{G_{v}} Y_{v} \rightarrow Y$ as a natural homomorphism of $\Lambda(G)$-modules. By the universality of direct sum, we obtain the map $\bigoplus_{v \mid p} \operatorname{Ind}_{G}^{G_{v}} Y_{v} \rightarrow Y$. As $X \subset Y$ and $X_{v} \subset Y_{v}$, we also have its restriction $h: \bigoplus_{v \mid p} \operatorname{Ind}_{G}^{G_{v}} X_{v} \rightarrow X$. By this construction it is clear that $h$ is the dual of the standard localization map

$$
H^{1}\left(K_{S} / K, A(p)\right) \rightarrow \bigoplus \operatorname{Coind}_{G}^{G_{v}} H^{1}\left(K_{v, \infty}, A(p)\right) \rightarrow \bigoplus \operatorname{Coind}_{G}^{G_{v}} H^{1}\left(K_{v, \infty}, \widetilde{A}(p)\right) .
$$

Hence we obtain the following commutative diagram:

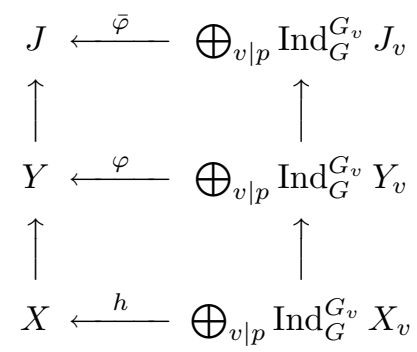

Here $\bar{\varphi}$ is the induced map from $\varphi$ so that the diagram is commutative. Taking the derived functor $\mathrm{E}^{i}$ associated to the contravariant functor $\operatorname{Hom}_{\Lambda}(\bullet, \Lambda)$ gives the diagram of the lemma. Its commutativity follows from the functoriality of the derived functor.

We have coker $(h)=\mathrm{E}^{2}(\mathfrak{X})$. This is because we have an exact sequence

$$
\mathrm{E}^{1}(X) \rightarrow \bigoplus_{v \mid p} \operatorname{Ind}_{G}^{G_{v}} \mathrm{E}_{v}^{1}\left(X_{v}\right) \rightarrow \mathrm{E}^{2}(\mathfrak{X}) \rightarrow \mathrm{E}^{2}(X) .
$$

But $\mathrm{E}^{2}(X)=0$ by the assumption.

Lemma 4. The cokernel of $\psi$ in the diagram of the Lemma 3 is a finitely generated $\mathbb{Z}_{p}$-module.

Proof. To see this, first we note that since $H^{2}\left(K_{S} / K_{\infty}, A(p)\right)=0$,

$$
\lim _{n} H^{2}\left(K_{S} / K_{n}, T_{p} A\right)
$$

is a torsion $\Lambda$-module (OV2 3.3). Hence by OV1, 4.10, we have

$$
\mathrm{E}^{1}(Y)=\lim _{n} H^{2}\left(K_{S} / K_{n}, T_{p} A\right)
$$

It is known from the Tate-Poitou sequence ([NSW] $)$ that

$$
\mathrm{E}^{1}\left(Y_{v}\right)=\lim _{n} H^{2}\left(K_{v, n}, T_{p} \widetilde{A}\right)
$$

is isomorphic to the dual of the kernel of the reduction map

$$
\hat{A}(p)\left(K_{v, \infty}\right) \rightarrow \tilde{\hat{A}}(p)\left(K_{v, \infty}\right) .
$$

From what we have seen in the proof of Lemma 3, the homomorpshim $\psi$ in the diagram of Lemma 3 is obtained as the composite of $r$ and $q$ in the following 
diagram:

$$
\begin{aligned}
& \underset{\|}{\lim _{n}} H^{2}\left(K_{S} / K_{n}, T_{p} A\right) \stackrel{r}{\rightarrow} \bigoplus_{v \mid p} \operatorname{Ind}_{G}^{G_{v}} \underset{n}{\lim _{\downarrow}} H^{2}\left(K_{v, n}, T_{p} A\right) \\
& \varliminf_{n} H^{2}\left(K_{S} / K_{n}, T_{p} A\right) \quad \bigoplus_{v \mid p} \operatorname{Ind}_{G}^{G_{v}}{\underset{\leftarrow}{n}}_{\lim _{n}} H^{2}\left(K_{n, v}, T_{p} \widetilde{A}\right) \\
& \text { \| } \quad \mid \\
& \mathrm{E}^{1}(Y) \longrightarrow \bigoplus_{v \mid p} \operatorname{Ind}_{G}^{G_{v}} \mathrm{E}^{1}\left(Y_{v}\right)
\end{aligned}
$$

By the Tate-Poitou sequence, one sees that the cokernel of the map $r$ is $\hat{A}(p)\left(K_{\infty}\right)^{\vee}$. Hence the cokernel is finitely generated over $\mathbb{Z}_{p}$.

Let us put $D:=g\left(\mathrm{E}^{2}(J)\right)$ so that $\operatorname{coker}(g) \cong\left(\bigoplus_{v \mid p} \operatorname{Ind}_{G}^{G_{v}} \mathrm{E}_{v}^{2}\left(J_{v}\right)\right) / D$. Since $D$ is the image of $\mathrm{E}^{2}(J) \cong \mathrm{E}^{3}\left(A(p)^{\vee}\right)$ which is zero if $\operatorname{dim}(G) \neq 3$ and a free $\mathbb{Z}_{p}$-module of finite rank if $\operatorname{dim}(G)=3$ (Lemma 2), $D$ is a finitely generated $\mathbb{Z}_{p}$-module. We have the following exact sequence:

$$
0 \rightarrow D \rightarrow \bigoplus_{v \mid p} \operatorname{Ind}_{G}^{G_{v}} \mathrm{E}^{2}(J) \rightarrow\left(\bigoplus_{v \mid p} \operatorname{Ind}_{G}^{G_{v}} \mathrm{E}^{2}\left(J_{v}\right)\right) / D \rightarrow 0 .
$$

We first show that $\mathrm{E}^{2}\left(\left(\bigoplus_{v \mid p} \operatorname{Ind}_{G}^{G_{v}} \mathrm{E}^{2}\left(J_{v}\right)\right) / D\right)=0$. Since $D$ is a finitely generated $\mathbb{Z}_{p}$-module, $\mathrm{E}^{1}(D)=0$ as $\operatorname{dim}(G) \geq 3$. We also have

$$
\mathrm{E}^{2}\left(\bigoplus_{v \mid p} \operatorname{Ind}_{G}^{G_{v}} \mathrm{E}^{2}\left(J_{v}\right)\right)=\mathrm{E}^{2}\left(\bigoplus_{v \mid p} \operatorname{Ind}_{G}^{G_{v}} \mathrm{E}^{3}\left(\widetilde{A}(p)^{\vee}\right)\right)=\bigoplus_{v \mid p} \operatorname{Ind}_{G}^{G_{v}} \mathrm{E}^{2} \mathrm{E}^{3}\left(\widetilde{A}(p)^{\vee}\right)=0 .
$$

(It can be directly seen that $\mathrm{E}^{2} \mathrm{E}^{3}\left(\widetilde{A}(p)^{\vee}\right)=0$. Or, in general, $\mathrm{E}^{i} \mathrm{E}^{j}(M)=0$ if $i<j$ for any finitely generated $\Lambda$-module $M$.) Hence $\mathrm{E}^{2}\left(\left(\bigoplus_{v \mid p} \operatorname{Ind}_{G}^{G_{v}} \mathrm{E}^{2}\left(J_{v}\right)\right) / D\right)=0$.

Let us now consider the following exact sequence:

$$
0 \rightarrow C \rightarrow \mathrm{E}^{2}(\mathfrak{X}) \rightarrow\left(\bigoplus_{v \mid p} \operatorname{Ind}_{G}^{G_{v}} \mathrm{E}^{2}\left(J_{v}\right)\right) / D \rightarrow 0
$$

where $C=\operatorname{ker}(s)$ is a quotient of $B:=\operatorname{coker}(\psi)$. Hence $C$ is a finitely generated $\mathbb{Z}_{p}$-module as $B$ is. It induces an exact sequence

$$
\mathrm{E}^{2}\left(\left(\bigoplus_{v \mid p} \operatorname{Ind}_{G}^{G_{v}} \mathrm{E}^{2}\left(J_{v}\right)\right) / D\right) \rightarrow \mathrm{E}^{2} \mathrm{E}^{2}(\mathfrak{X}) \rightarrow \mathrm{E}^{2}(C) .
$$

But $\mathrm{E}^{2}\left(\left(\bigoplus_{v \mid p} \operatorname{Ind}_{G}^{G_{v}} \mathrm{E}^{2}\left(J_{v}\right)\right) / D\right)=0$ as we have seen as above, and $\mathrm{E}^{2}(C)=0$ since $C$ is a finitely generated $\mathbb{Z}_{p}$-module and $\operatorname{dim}(G) \geq 3$. Thus we have obtained $\mathrm{E}^{2} \mathrm{E}^{2}(\mathfrak{X})=0$.

Now only the case $\mathrm{E}^{2}(X) \neq 0$ remains to be checked (i.e. the $\operatorname{dim}(G)=4$ case). Remember that $\mathrm{E}^{2}(X)$ is a free $\mathbb{Z}_{p}$-module of finite rank (Lemma 2 ). We have the following exact sequence:

$$
\mathrm{E}^{1}(X) \rightarrow^{h} \bigoplus_{v \mid p} \operatorname{Ind}_{G}^{G_{v}} \mathrm{E}_{v}^{1}\left(X_{v}\right) \rightarrow \mathrm{E}^{2}(\mathfrak{X}) \rightarrow \mathrm{E}^{2}(X) .
$$


Therefore if we write $M:=\operatorname{coker}(h)$, then we get a short exact sequence:

$$
0 \rightarrow M \rightarrow \mathrm{E}^{2}(\mathfrak{X}) \rightarrow T \rightarrow 0,
$$

where $T$ is a free $\mathbb{Z}_{p}$-module contained in $\mathrm{E}^{2}(X)$.

We have had the argument above to show that $\mathrm{E}^{2}(\mathfrak{X})=0$ assuming $\mathrm{E}^{2}(X)=0$. But it has actually shown that $\mathrm{E}^{2}(\operatorname{coker}(h))=0$ without the assumption $\mathrm{E}^{2}(X)=$ 0 . (If $\mathrm{E}^{2}(X)=0$, then $\mathrm{E}^{2}(\mathfrak{X})=\operatorname{coker}(h)$.) Therefore in the present case we have $\mathrm{E}^{2}(M)=0$.

The short exact sequence (5) gives the following exact sequence:

$$
\mathrm{E}^{2}(T) \rightarrow \mathrm{E}^{2} \mathrm{E}^{2}(\mathfrak{X}) \rightarrow \mathrm{E}^{2}(M)=0 .
$$

But $\mathrm{E}^{2}(T)=0$ by Lemma 2. Thus we get $\mathrm{E}^{2} \mathrm{E}^{2}(\mathfrak{X})=0$. This finishes the proof of the theorem.

\section{ACKNowledgements}

The author is very grateful to Yuichiro Taguchi and to the referee. The author was supported by Korea Institute for Advanced Study (KIAS).

\section{REFERENCES}

[CG] J. Coates and R. Greenberg, Kummer theory of abelian varieties over local fields, Invent. Math. 124 (1996), 129-174. MR1369413 (97b:11079)

[Gr] R. Greenberg, The structure of Selmer groups, Proceedings Natl. Acad. of Science 94 (1997), 11125-11128. MR 1491971 (98m:11123)

[Ma] K. Matsuno, Finite $\Lambda$-submodules of Selmer groups of abelian varieties over cyclotomic $\mathbb{Z}_{p}$-extensions, Journal of Number Theory 99 (2003), 415-443. MR.1969183(2004c:11098)

[Ja] U. Jannsen, Iwasawa modules up to isomorphism, Advanced Studies in Pure Mathematics, vol. 17, Algebraic Number Theory, in honour of K. Iwasawa, pp. 171-207, 1989. MR1097615 (93c:11095)

[NSW] J. Neukirch, A. Schmidt, and K. Wingberg, Cohomology of Number Fields, Springer, 2000. MR.1737196 (2000j:11168)

[OV1] Y. Ochi and O. Venjakob, On the structure of Selmer groups over p-adic Lie extensions, Journal of Algebraic Geometry 11 (2002), 547-580. MR1894938(2003m:11082)

[OV2] Y. Ochi and O. Venjakov, On the ranks of Iwasawa modules over p-adic Lie extensions, Math. Proc. Camb. Phil. Soc. 135 (2003), 25-43. MR.1990830(2004d:11107)

[Pe] B. Perrin-Riou, Groupe de Selmer d'une courbe elliptique a multiplication complexe, Compo. Math., 43 (1981), 387-417. MR0632436 (83i:14031)

[Ri] K. Ribet, Division fields of abelian varieties with complex multiplication, Memoires de la SMF 1980, pp. 75-94. MR0608640 (83e:14029a)

[Se] J-P. Serre, Collected Papers IV, Springer. MR.1730973 (2001e:01037)

[ST] J-P. Serre and J. Tate, Good reduction of abelian varieties, Ann. of Math. 88 (1968), 492-517. MR0236190(38:4488)

[Ve] O. Venjakob, On the structure theory of the Iwasawa algebra of a p-adic Lie group, J. Eur. Math. Soc. (JEMS) 4 (2002), no. 3, 271-311. MR1924402 (2004h:16029)

School of Science and Engineering, Tokyo Denki University, Tokyo, 101-8457, Japan

E-mail address: ochi@u.dendai.ac.jp 